\title{
Correction to: A polyhedron comparison theorem for 3-manifolds with positive scalar curvature
}

\author{
Chao Li ${ }^{1}$
}

Published online: 8 February 2022

(C) Springer-Verlag GmbH Germany, part of Springer Nature 2022

Correction to: Invent. math. (2020) 219:1-37

$$
\text { https://doi.org/10.1007/s00222-019-00895-0 }
$$

We use the notations from [Li20] in this erratum. There is a mistake in the proof of Lemma 3.1 of of [Li20], affecting Theorem 1.4 and Theorem 1.5 of the paper. Indeed, the assumption that $\measuredangle\left(F_{j, \infty}, F_{j+1, \infty}\right)<\measuredangle\left(F_{j}^{\prime}, F_{j+1}^{\prime}\right)$ does not guarantee that $T_{p} M$ can be placed strictly within the tangent cone of $P$ at its vertex. It turns out that Lemma 3.1 requires assumptions (1.2) and (1.3), which we state here. Let $P \subset \mathbf{R}^{3}$ be a cone type polyhedron with a vertex $p^{\prime}$, side faces $F_{j}^{\prime}$ and base face $B^{\prime}$, and $\gamma_{j}$ be the dihedral angle between $F_{j}^{\prime}$ and $B^{\prime}$. In the sequel we allow triangles to be degenerate, namely two sides of a triangle can be parallel-in which case the angle between those two sides is zero.

$$
\gamma_{j} \leq \pi / 2, j=1,2, \cdots, k
$$

The online version of the original article can be found under https://doi.org/10.1007/s00222019-00895-0.

Chao Li

chaoli@nyu.edu

1 Department of Mathematics, Stanford University, Stanford, USA 
On $B^{\prime}$, there exists a vertex $A^{\prime}$ such that the following holds: let $A_{1}^{\prime}, A_{2}^{\prime}$ be the adjacent vertices of $A^{\prime}$. Then for all sides $L^{\prime}$ of $B^{\prime}$ not containing $A^{\prime}$, the line through $L^{\prime}$ forms a triangle (or a degenerate triangle) $\Delta$ with the rays given by $\overline{A^{\prime} A_{1}^{\prime}}$ and $\overline{A^{\prime} A_{2}^{\prime}}$, such that inner angles of $\Delta$, except the one at $A^{\prime}$, are not larger than $\pi / 2$.

Remark 0.1 Assumption 1.3 is satisfied by all triangles. In general, it is equivalent to to the existence of three consecutive vertices $A_{1}^{\prime}, A^{\prime}, A_{2}^{\prime}$, at which the inner angles satisfy:

$\measuredangle A_{1}^{\prime} \leq \frac{\pi}{2}, \quad, \measuredangle A_{2}^{\prime} \leq \frac{\pi}{2}, \quad \frac{\pi}{2} \leq \measuredangle A^{\prime}+\measuredangle A_{1}^{\prime} \leq \pi, \quad \frac{\pi}{2} \leq \measuredangle A^{\prime}+\measuredangle A_{2}^{\prime} \leq \pi$.

Lemma 3.1 Let $P \subset \mathbf{R}^{3}$ be a polyhedron of cone type, $(M, g)$ be of $P$-type. Assume $P$ satisfies (1.2) and (1.3), and $\measuredangle_{i j}(M)<\measuredangle_{i j}(P)$. Then the infimum $\mathcal{I}$ appeared in (1.4) is negative.

Proof Consider the tangent cone of $M$ at the vertex $p$, and the side faces $F_{j, \infty}$. Denote by $F_{j}^{\prime}$ the side faces of $P$. In the base face of $P$, take the vertex $A^{\prime}$ satisfying (1.3), and without loss of generality assume $A^{\prime}$ lies on the intersection of $F_{1}^{\prime}, F_{2}^{\prime}$. Take $A \in F_{1, \infty} \cap F_{2, \infty}, A \neq p$. Take a plane $\pi \subset \mathbf{R}^{3}$ through $A$, such that the dihedral angle between $\pi$ and $F_{j, \infty}$ equals $\gamma_{j}, j=1,2$ (such a plane $\pi$ exists since $M$ satisfies assumption (1.1)). Denote by $E_{\infty} \subset T_{p} M$ the compact closed region separated by $\pi$. We will prove that for any $l \geq 3$, the dihedral angle between $F_{l, \infty}$ and $\pi$ is strictly greater than $\gamma_{l}$, and thus

$$
\mathcal{H}^{2}\left(\pi \cap \partial E_{\infty}\right)-\sum_{j=1}^{k}\left(\cos \gamma_{j}\right) \mathcal{H}^{2}\left(F_{j, \infty} \cap \partial E_{\infty}\right)<0,
$$

finishing the proof.

For simplicity of notations we prove the statement for $l=3$. (In general, take a face $F_{j, \infty}$ and consider the cone formed by the planes through $F_{1, \infty}, F_{2, \infty}, F_{j, \infty}$, and compare to the the cone formed by the planes through $F_{1}^{\prime}, F_{2}^{\prime}, F_{j}^{\prime}$ with exactly the same argument.) We fix some notations. Denote by $B=F_{1, \infty} \cap \pi \cap F_{3, \infty}, C=F_{2, \infty} \cap \pi \cap F_{3, \infty}, v$ the unit normal vector of $\pi$ pointing to $p$. For $j=1,2,3$, denote by $v_{j}$ the unit normal vector of $F_{j, \infty}$ pointing out of $T_{p} M, \beta_{j}=\measuredangle\left(\nu, v_{j}\right)$ (thus $\beta_{1}=\gamma_{1}, \beta_{2}=\gamma_{2}$ ), and $\alpha_{1}=\measuredangle(\overrightarrow{A B}, \overrightarrow{A C}), \alpha_{2}=\measuredangle(\overrightarrow{B A}, \overrightarrow{B C}), \alpha_{3}=\measuredangle(\overrightarrow{C A}, \overrightarrow{C B})$. Denote by $\theta$ the dihedral angle between $F_{1, \infty}, F_{2, \infty}$, and $\theta_{j}$ the dihedral angle between $F_{3, \infty}$ and $F_{j, \infty}, j=1,2$. We then denote the corresponding geometric quantities 
in $P$ by $v^{\prime}, v_{1}^{\prime}$, etc. In particular, since the side faces of $P$ meet the base face at $\gamma_{j}$, we have $\beta_{j}^{\prime}=\gamma_{j}, j=1,2,3$.

By the spherical law of cosines, applied to the spherical triangle cut out by $E_{\infty}$ from a small 2-sphere centered at $A$, we have

$$
\begin{aligned}
\cos \alpha_{1} \sin \beta_{1} \sin \beta_{2} & =\left(v \times v_{1}\right) \cdot\left(v_{2} \times v\right)=\left(v \cdot v_{2}\right)\left(v_{1} \cdot v\right)-v_{1} \cdot v_{2} \\
& =\cos \beta_{1} \cos \beta_{2}+\cos \theta .
\end{aligned}
$$

And the similar equation holds on $P$. Since $\beta_{1}=\beta_{1}^{\prime}, \beta_{2}=\beta_{2}^{\prime}, \theta<\theta^{\prime}$, we have $\alpha_{1}<\alpha_{1}^{\prime}$. As $\sum_{j=1}^{3} \alpha_{j}=\sum_{j=1}^{3} \alpha_{j}^{\prime}=\pi$, we have either $\alpha_{2}>\alpha_{2}^{\prime}$ or $\alpha_{3}>\alpha_{3}^{\prime}$. Assume that $\alpha_{2}>\alpha_{2}^{\prime}$. Analogous to (0.1), we have

$$
\begin{aligned}
& \cos \alpha_{2} \sin \beta_{2} \sin \beta_{3}=\cos \beta_{2} \cos \beta_{3}+\cos \theta_{1}, \\
& \cos \alpha_{2}^{\prime} \sin \beta_{2}^{\prime} \sin \beta_{3}^{\prime}=\cos \beta_{2}^{\prime} \cos \beta_{3}^{\prime}+\cos \theta_{1}^{\prime} .
\end{aligned}
$$

By assumption, $\theta_{1}<\theta_{1}^{\prime}$. Hence

$$
\cos \alpha_{2} \sin \beta_{2} \sin \beta_{3}-\cos \beta_{2} \cos \beta_{3}>\cos \alpha_{2}^{\prime} \sin \beta_{2}^{\prime} \sin \beta_{3}^{\prime}-\cos \beta_{2}^{\prime} \cos \beta_{3}^{\prime} \text {. }
$$

Note that $\cos \beta_{2}^{\prime}=\cos \beta_{2} \geq 0$ (by (1.2)). We claim that (0.2) implies that $\beta_{3}>\beta_{3}^{\prime}$ as follows. Suppose the contrary, that $\beta_{3} \leq \beta_{3}^{\prime}$. Then $\cos \beta_{2} \cos \beta_{3} \geq$ $\cos \beta_{2}^{\prime} \cos \beta_{3}^{\prime}$. Thus (0.2) implies that $\cos \alpha_{2} \sin \beta_{3}>\cos \alpha_{2}^{\prime} \sin \beta_{3}^{\prime}$. However, (1.3) implies $\alpha_{2}^{\prime} \leq \pi / 2 \Rightarrow \cos \alpha_{2}^{\prime} \geq 0$. This is a contradiction.

We now reformulate the main theorems of the paper. Other steps of their proofs are unchanged.

Theorem 1.4 Let $\left(M^{3}, g\right)$ be a Riemannian polyhedron of P-type with side faces $F_{1}, \cdots, F_{k}$, where $P \subset \mathbf{R}^{3}$ is a cone or prism with side faces $F_{1}^{\prime}, \cdots, F_{k}^{\prime}$. Denote by $\gamma_{j}$ the angle between $F_{j}^{\prime}$ and the base face of $P$. Assume that everywhere along $F_{j} \cap F_{j+1}$,

$$
\left|\pi-\left(\gamma_{j}+\gamma_{j+1}\right)\right|<\measuredangle\left(F_{j}, F_{j+1}\right),
$$

and when $P$ is a cone, $P$ satisfies (1.2) and (1.3).

Then the strict comparison statement holds for $(M, g)$. Namely, if $R(g) \geq$ 0 , and each $F_{j}$ is mean convex, then the dihedral angles of $M$ cannot be everywhere less than those of $P$.

Theorem 1.5 Under the same assumptions of Theorem 1.4 and the extra assumption (when $P$ is a prism) that

$$
\gamma_{j} \leq \pi / 2, j=1,2, \cdots, k, \quad \text { or } \gamma_{j} \geq \pi / 2, j=1,2, \cdots, k,
$$


then we have the rigidity statement. Namely, if $R(g) \geq 0$, each $F_{j}$ is mean convex, and $\measuredangle_{i j}(M, g) \leq \measuredangle_{i j}\left(P, g_{\text {Euclid }}\right)$, then $(M, g)$ is isometric to a flat polyhedron in $\mathbf{R}^{3}$.

Acknowledgements The author wishes to thank Bernhard Hanke for pointing out the mistake in Lemma 3.1.

\section{Reference}

[Li20] Li, C.: A polyhedron comparison theorem for 3-manifolds with positive scalar curvature. Invent. Math. 219, 1-37 (2020)

Publisher's Note Springer Nature remains neutral with regard to jurisdictional claims in published maps and institutional affiliations. 